\title{
Quantitative assessment of smooth-pursuit eye movements in healthy and epileptic subjects
}

\author{
P R M B ITTENCOURT, M A GRESTY, * A N A R I CHENS \\ From the Clinical Pharmacology Unit, University Department of Clinical Neurology \\ and MRC Hearing and Balance Unit, * Institute of Neurology, The National Hospital, London
}

SUMMARY A quantitative electro-oculographic method was used to assess the impairment in smooth-pursuit and vestibulo-ocular reflex suppression eye movements in 12 epileptic patients and eight normal volunteers. Both types of eye movement were impaired by a factor of $40 \%$ in the epileptic patients, and the impairment was at least partly due to the treatment with phenytoin and phenobarbitone. There was a strong positive correlation between the performances in vestibulo-ocular reflex suppression and smooth-pursuit.

Among the more prominent signs of acute antiepileptic drug toxicity are those of vestibulocerebellar origin, such as nystagmus, ataxia and dysarthria. Less commonly reported, perhaps because they are more subtle, are slowness of thought, asterixis and behavioural changes. ${ }^{12}$ Impairment of brain function in patients who do not show evident signs of acute antiepileptic drug toxicity, and whose serum antiepileptic drug concentrations are within the so-called therapeutic ranges, ${ }^{3}$ is more difficult to measure, but needs to be assessed in determining the long-term effects of the treatment, as well as of the seizure disorder itself. In this study we have established simple and reliable electro-oculographic criteria to indicate the degree of impairment in oculomotor function in drug-treated epileptic patients who do not show evident signs of acute antiepileptic drug toxicity.

All eye movements except microtremor either switch gaze from one target to the next, or maintain gaze when either the target or the patient's head is moving. Saccades are the fast movements used to transfer the fixation point between various targets. Their peak velocity may reach $700^{\circ} / \mathrm{s},{ }^{4}$ and vision is not optimum during the saccade itself. ${ }^{\overline{ }}$ The vestibulo-ocular reflex (VOR) is used to maintain gaze on stationary targets while the head is moving. It generates eye movements of equal speed but opposite direction to the head

\footnotetext{
Address for reprint requests: Dr PRM Bittencourt, Clinical Pharmacology Unit, Institute of Neurology, The National Hospital, Queen Square, London WCIN 3BG.

Accepted 30 July 1980
}

movement, such that head and eye displacements cancel each other and the direction of visual fixation remains undisturbed. ${ }^{6}$ Smooth-pursuit eye movements are used to track targets moving in the external world when the head is immobile. Should a person try to track a moving object using both head and eyes, the VOR and the pursuit system come into conflict. As the head moves in the direction of the target, the VOR drives the eyes in the opposite direction, while the pursuit system is trying to drive the eyes along with the target. ${ }^{7}$ The VOR then, has to be overcome, so that the added head and eye velocity can equal that of the moving target. The mechanism by which the VOR is overcome is referred to as VOR suppression. There is much evidence that an alternative role for the pursuit system is VOR suppression. ${ }^{7-9}$ An important finding is that there are limits to the velocity of both smooth-pursuit and VOR suppression. When the head is immobile, the pursuit system can track moving targets up to a certain threshold velocity, above which saccades have to be used to catch up with the target. If the head is moving, head velocity may exceed the capacity of the pursuit system, so that complete supression is impossible. It has been suggested that when pursuit reaches its threshold velocity, so does VOR suppression. ${ }^{7-9}$ Depending on experimental conditions, the pursuit threshold velocity in the human has been given as between $40-80^{\circ} / \mathrm{s}^{9}{ }^{10}$ The VOR suppression threshold velocity seems to be similar, in a wide variety of neurological patients and in normal volunteers. ${ }^{\top 9}$ 
In this study we have used pursuit and VOR suppression threshold velocities as indices of oculomotor function in order to look for abnormalities in drug-treated epileptic pains.

\section{Subjects}

Twelve adult drug-treated epilepic patients and eight healthy drug-free volunteers matched for age and sex were tested repeatedly over a period of six months. Patients were attending outpatient clinics, usually for changes aiming at optimum seizure control. Signs of other active medical or psychiatric disorders were absent, and they were not on centrally-acting drugs apart from antiepileptic drugs. None of the patients had evident signs of acute antiepilepiic drug toxicity, such as unextinguishable nystagmus, dysarthria, ataxia, asterixis, involuntary movements, slowness of thought or behavioural changes. ${ }^{12}$ The mean age of the patients was $24 \cdot 4( \pm 6 \cdot 6 \mathrm{SD})$ years, the mean duration of their epilepsy was 16.9 ( $\pm 7.5 \mathrm{SD})$ years and their mean IQ was $88.3( \pm 12 \mathrm{SD})$ as measured on the WAIS. The control subjects had a mean age of $28.2( \pm 7.2 \mathrm{SD})$ years, and comprised medical and paramedical staff. During the course of the study, five patients had complex partial seizures, three had complex partial seizures with secondary generalisation, two had primary generalised tonic-clonic seizures, one had myoclcnic jerks and ore was seizurc-írce.

\section{Methods}

Epileptic patients and normal volunteers were tested two to four times with intervals of four to six weeks between tests. All tests were conducted in constant ambient illumination of dark red light, in a sound attenuated laboratory, at approximately the same time of day. Eye movements were recorded by direct-coupled electro-oculography, displayed on a rectilinear electrostatic ElemaSchonnander chart recorder. Stick-on silver-silver chloride electrodes were placed lateral to both outer canthi, with a similar reference electrode on the forehead.

Smooth-pursuit, VOR and VOR suppression were recorded in the same session, lasting approximately 10 minutes. Subjects sat in a chair free to rotate about its vertical axis, corresponding to the body's longitudinal axis, with the head restrained by a head rest. For measurement of VOR the chair was oscillated sinusoidally, while subjects tried to maintain gaze on an immobile target, $2 \mathrm{~m}$ from the nasion. For measurement of VOR suppression, a $10 \mathrm{~mm}$ light bulb was attached to the chair, $370 \mathrm{~mm}$ from the nasion. The chair and the light bulb were oscillated sinusoidally while subjects tried to maintain gaze on the light bulb. For recording smooth-pursuit the chair was kept stationary and subjects were asked to follow a point source of light moving sinusoidally on a $650 \mathrm{~mm}$-wide Lanelec oscilloscope, placed $650 \mathrm{~mm}$ from the nasion. Both target and chair velocity were varied between 9.4 and $94 \%$ s. Target and chair motion had a peak-to-peak amplitude of $30^{\circ}$. Calibration of chair, target and eye displacement signals were made before and after each test. Blood samples were taken at the time of testing, and the serum kept for subsequent determination of serum antiepileptic drug concentrations by gas-chromatography (phenytoin and phenobarbitone) or enzyme immuno-assay (carbamazepine).

\section{Visual analysis of records}

When the pursuit system is not able to keep up with increasing target velocities, an error signal (proportional to the retinal slip) is generated between target and eye position. ${ }^{6}$ Saccades are then used to catch up with the target, and their size increases as the gap between target and eye velocity widens (fig 1). Likewise, when suppression of the VOR is incomplete, saccades (part of a nystagmus-like trace, fig 2) are used to bring the eyes quickly on to the target. These saccades also increase in size as chair velocity (in phase with head and target) increases. In some cases, the VOR itself is not suppressed, and may underlie the nystagmus-like trace (fig 2), but in all cases the nystagmus was the first manifestation of failure of suppression.

We defined the "pursuit threshold velocity" as that peak angular velocity of target motion at which saccades larger than $5^{\circ}$ appeared consistently on the electro-oculogram. The "VOR suppression threshold velocity" was defined as the peak angular velocity of chair (head) motion at which $2^{\circ}$ saccades were consistently present on the electro-oculogram. The development of these criteria took into account the biological and electrogenic noise levels in our recordings, as well as the facility with which the naked eye can see these saccades. During smooth-pursuit, saccades larger than $15^{\circ}$ in amplitude, or those not in the direction of the target were not taken into account, since they usually demonstrated lack of attention, as described in psychiatric populations. ${ }^{112}$ 


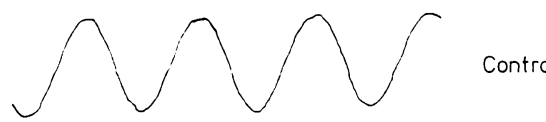

Control

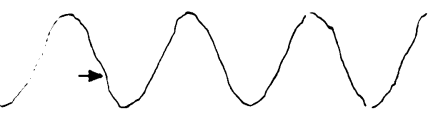

Epileptic

$38 \%$ s

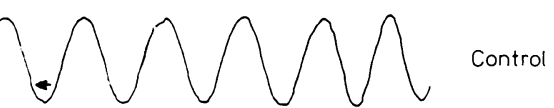

$56 \%$

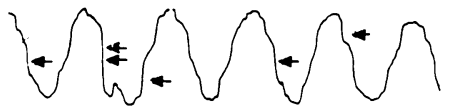

Epileptic

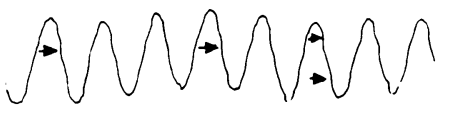

Control

$85 \% \mathrm{~s}$

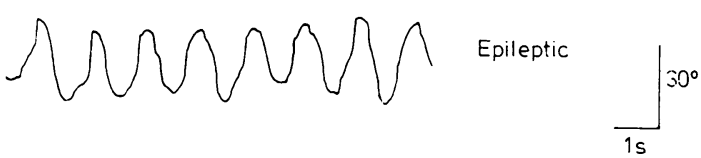

Fig 1 Kaw smooth-pursuit records of control and epileptic subjects. Peak angular velocity calculated by $2 \pi F A$, where $F$ is the frequency of sinusoidal oscillation and $A$ is half of the total amplitude of excursion. At $38^{\circ} / \mathrm{s}$ the epileptic subject shows sma!l saccades, never larger than $3^{\circ}(\rightarrow)$. At $56^{\circ} / \mathrm{s}$ the control subject starts to show similar saccades, while the epileptic subject produces saccades consistently larger than $5^{\circ}(\leftarrow)$. At $85^{\circ} / \mathrm{s}$ the epileptic patient cannot follow, the total amplitude of his eye movement being decreased to $20^{\circ}$. The control subject has started using saccades of about $5^{\circ}$. (६) shows an inattention saccade $\left(24^{\circ}\right.$ in amplitude).

\section{Results}

The mean number of antiepileptic drugs per patient was $1 \cdot 75$. Figure 3 shows the range of phenytoin, phenobarbitone and carbamazepine levels observed during the study. Three of the patients had "toxic" serum concentrations of phenytoin, and one of phenobarbitone, when first tested. In all four patients the levels returned to the "therapeutic" range as the study progressed. In one patient phenytoin was withdrawn, and, in another, primidone. Both remained on carbamazepine. The six other patients had serum levels that never exceeded the upper limits of the therapeutic range, and none had drugs withdrawn during the study.

VOR was not impaired in any of the subjects, both the amplitude and morphology of the eye movement traces remaining unchanged throughout the range of velocities examined. For smoothpursuit and VOR suppression a very different picture emerged (fig 4). The mean ( \pm SD) smoothpursuit threshold velocities were $78 \pm 13 \%$ s in the control group and $48 \pm 13 \% / \mathrm{s}$ in the epileptic patients. The VOR suppression threshold velocities were $64 \pm 14 \% / \mathrm{s}$ in the controls and $39 \pm 11.5 \% / \mathrm{s}$ in the epileptic subjects. Both were significantly higher in the control group ( $p<0 \cdot 01$, Student's test for unpaired observations). The differences remained significant if those results obtained from patients with high serum antiepileptic drug concentrations (see below) were excluded. The mean
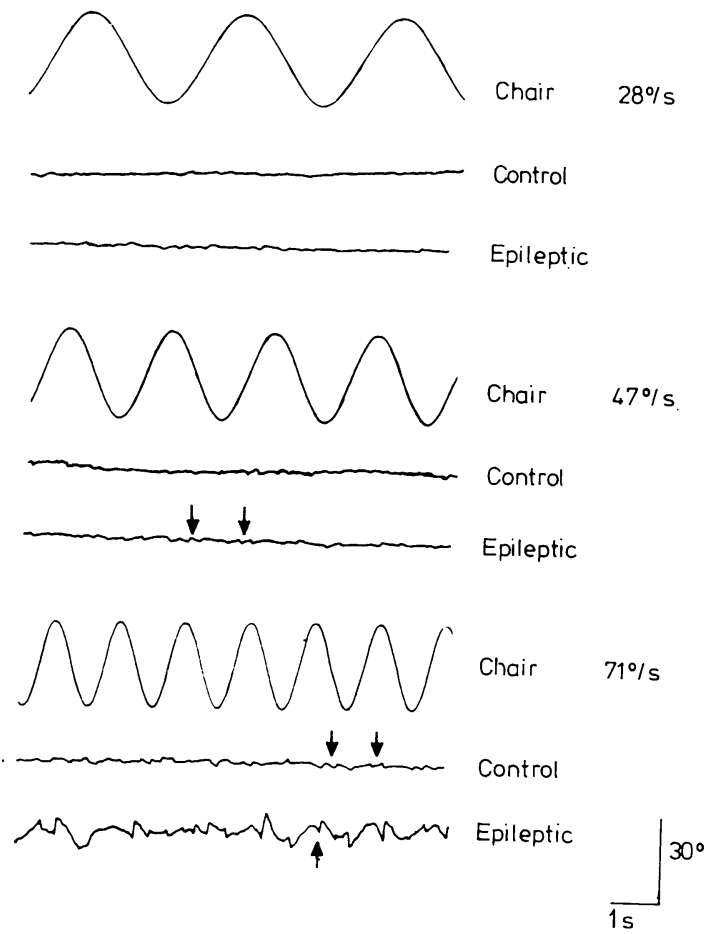

Fig 2 Raw VOR suppression records of control and epileptic subjects. Chair peak angular velocity calculated by $2 \pi F A$, where $F$ is the frequency of sinusoidal oscillation and $A$ is half of the total angular excursion of chair motion. At $28^{\circ} / \mathrm{s}$ both subjects are able to keep their eyes on target, which is being oscillated in phase with chair and head, although the control subject does so more accurately. At $47^{\circ}$ s the control subject shows ill-defined oscillations of the eye movement trace, while the epileptic subject shows a clear nystagmus-like pattern ( $\downarrow)$, with saccades of approximately $2^{\circ}$ in amplitude. At $71^{\circ} / \mathrm{s}$ the control produces a similar pattern, while the epileptic patient show's very large saccades superimposed on a VOR-like trace $(\uparrow)$. 


\section{$\underline{\mathrm{CBZ}}$}

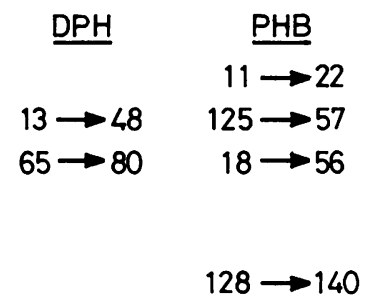

MA

CS

FM

JD

JS

$\mathrm{AH}$

$$
\begin{aligned}
106 & \rightarrow 41 \\
67 & \rightarrow 39 \\
214 & \rightarrow 46 \\
110 & \rightarrow 74 \\
56 & \rightarrow 0
\end{aligned}
$$$$
31 \rightarrow 38
$$

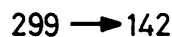

$127 \rightarrow 0$

Fig 3 Range of carbamazepine (CBZ), phenytoin $(D P H)$ and phenobarbitone $(P H B)$ serum concentrations found in the epileptic patients while taking part in the study. JA was also on sodium valproate (1200 $\mathrm{mg}$ daily) and FM on ethosuximide (750 mg daily) throughout the study.

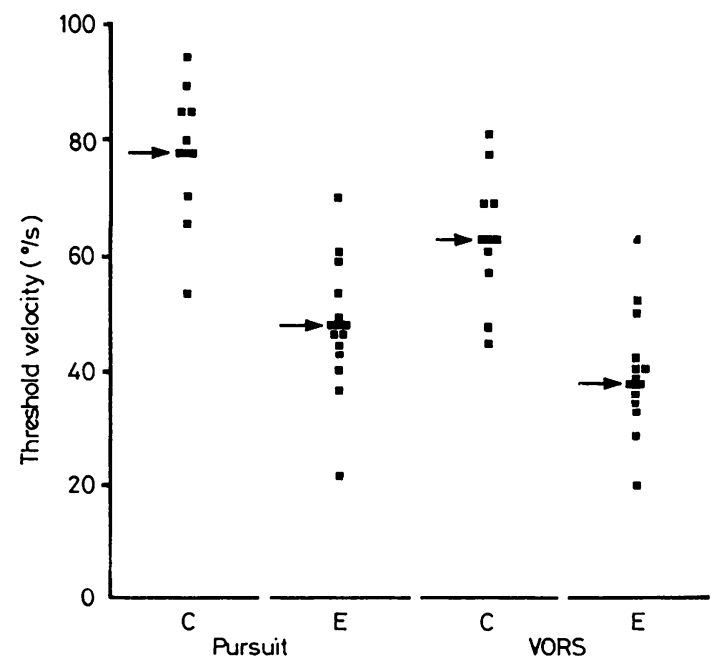

Fig 4 Smooth-pursutt (pursuit) and VOR suppression $(V O R S)$ threshold velocities in 12 epileptic $(E)$ and 8 control $(C)$ subjects. Values expressed are means of all performances taken together for each of the subjects. $(\rightarrow \square)$ shows the mean thresholds in each test.

smooth-pursuit threshold in the epileptic group was $60 \%$ of that of the controls, while VOR suppression was $61 \%$. The overlap between the two populations was between approximately $50-70 \% / \mathrm{s}$ in the case of smooth-pursuit and between 40$60 \% / \mathrm{s}$ for VOR suppression. Only one of the con-

trols had a smooth-pursuit threshold of less than $60^{\circ} / \mathrm{s}$ while only two of the epileptic subjects had results above that value.

Regression analysis showed a highly significant positive linear correlation between smoothpursuit and VOR threshold velocities (fig 5). Throughout the range examined, smooth-pursuit tended to have a slightly higher threshold (approximately $10^{\circ} / \mathrm{s}$ ) than VOR suppression, but there was no significant difference between the two eye movement thresholds in either of the two groups.

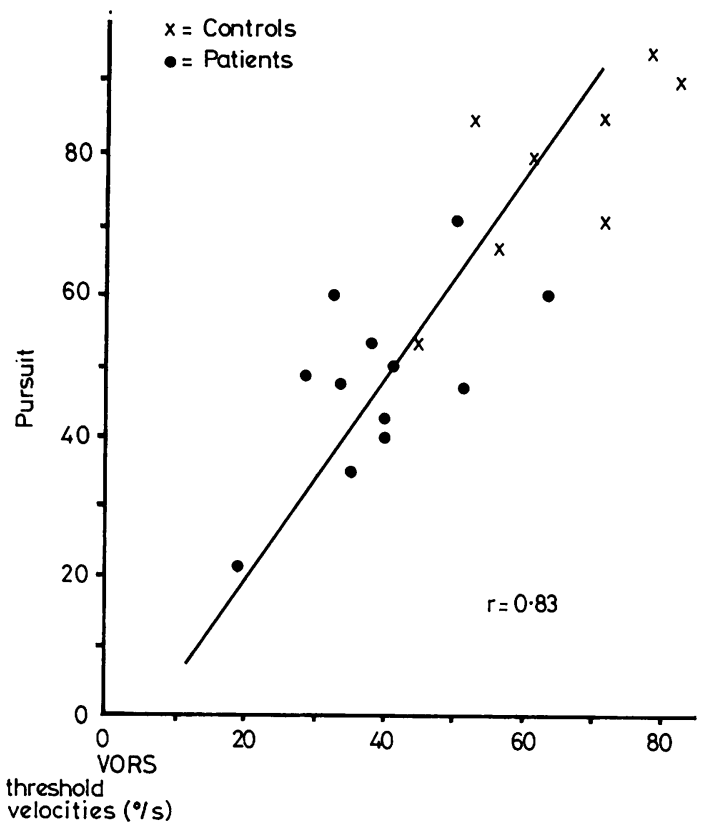

Fig 5 Positive linear correlation $(r=0.83, p<0.01)$ between smooth-pursuit and VOR suppression (VORS) threshold velocities in the 20 subjects studied. $V$ alues plotted are those of fig $4(\bullet=$ epileptic and $x=$ control subjects).

Four of the epileptic patients entered the study with high serum levels of phenytoin (MA, FM, JD) or phenobarbitone (CS), and subsequently recovered. Two other patients had a drug withdrawn during the study, namely primidone (AH) and phenytoin (JS). These patients were considered to have had a "major" change in their drug treatment, which caused the serum phenytoin and phenobarbitone concentrations either to cross the upper limit of the therapeutic range or to fall to zero. The six other patients at no time had high serum antiepileptic drug concentrations, and did not have drugs withdrawn or added to their therapy. These were considered to have had 
"minor" changes in their treatment (fig 6). The mean ( \pm SD) smooth-pursuit threshold velocity increased from $34 \cdot 7 \pm 7 \cdot 6 \%$ s to $52 \cdot 2 \pm 12 \% / \mathrm{s}$ after major changes $(p<0.01$, Student's test for paired observations), while it did not alter in the minor change group, remaining at approximately $52 \% / \mathrm{s}$. There was no significant intersession change in performance in the control group.

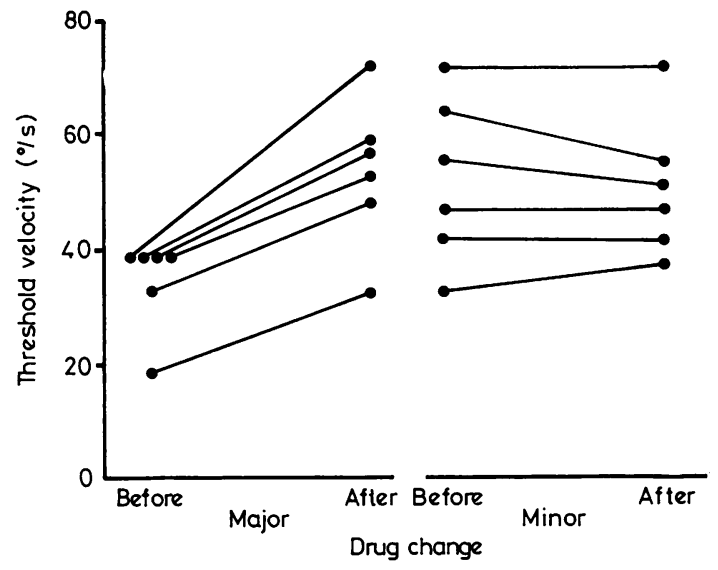

Fig 6 Effect of major and minor changes in phenytoin or phenobarbitone serum concentrations on the smooth-pursuit threshold velocity. Values expressed are results of one session, immediately before and after changes in drug treatment.

\section{Discussion}

Abnormalities of smooth-pursuit or VOR suppression eye movements in epileptic patients do not seem to have been described previously, although carbamazepine overdosage (more than $1600 \mathrm{mg}$ orally) produced a pronounced impairment in smooth-pursuit in three patients with trigeminal neuralgia. ${ }^{13}$ Phenytoin was reported to have impaired the response to a standard caloric test in an epileptic patient, who recovered when the dosage was decreased. ${ }^{14}$ Various centrally-acting drugs have been shown to impair smooth-pursuit eye movements, including alcohol, ${ }^{15}$ barbiturates ${ }^{16}$ and probably nitrazepam. ${ }^{17}$ Smooth-pursuit eye movements are impaired in patients with a variety of lesions at different levels of the central nervous system..$^{7} 18$

Visual suppression of the caloric induced vestibular nystagmus is the more commonly used test for differentiating central from peripheral lesions of the vestibulo-ocular reflex arc. ${ }^{19}$ Although failure of suppression has been more commonly described in patients with posterior fossa lesions, there is evidence that, like smooth-pursuit, visual suppression of vestibular-induced nystagmus can also be impaired in patients with hemisphere lesions. ${ }^{7819}$ Our finding that there is a strong correlation between smooth-pursuit and VOR suppression threshold velocities in epileptic and normal subjects lends objective, quantitative support to the close physiological association between these two types of eye movement, as suggested in other reports. ${ }^{7-9}$

Our results indicate that treated epileptic patients, even those lacking clinical or biochemical evidence of anticonvulsant toxicity, nevertheless have smooth-pursuit and VOR suppression impaired by a mean of $40 \%$. We have also shown that the impairment is at least partly due to the drug treatment, specifically phenytoin and phenobarbitone, because a marked improvement occurred following a major reduction in therapy. It is possible that impairment of visual-vestibular mechanisms in epileptic patients may affect to a substantial degree their performance in tasks like operating machinery or crossing streets. ${ }^{9}$ It is still unclear how much of the impairment may be due to the antiepileptic drugs directly, or to the cerebellar morphological changes found in patients with longstanding epilepsy. ${ }^{20}$ There is some debate whether these pathological findings may be a consequence of the treatment, particularly phenytoin, or of various factors associated with the seizures. ${ }^{21} 22$

Simple visual observation of smooth-pursuit eye movements can provide the clinician with valuable information. If the technique of assessment is standardised, graded observations can be made of a clinical sign that although not diagnostic per se, allows more objective assessment of patients suffering from epilepsy or other neurological disorders. The indices used in this study can be taken as a model for such a test. The examiner should move a target at a fixed distance from the patient's nasion $(65 \mathrm{~cm})$ and keep the amplitude of the movement to $30^{\circ}(35 \mathrm{~cm})$. We suggest three velocity steps: approximately $25 \%$ s one cycle every four seconds), $50 \% / \mathrm{s}$ (one cycle every two seconds) and $75 \%$ s (one and a half cycles every two seconds).

The authors thank Mr S Hebdige for measuring the serum antiepileptic drug concentrations, Drs $\mathbf{J}$ Oxley and $\mathbf{J}$ Laidlaw for allowing us to study patients under their care, and Dr JD Hood for advice. Dr PRM Bittencourt was supported by a Thorn Epilepsy Research Fellowship. 


\section{References}

1 Plaa GL. Acute toxicity of antiepileptic drugs. Epilepsia 1975; 16:183-91.

2 Trimble M, Reynolds EH. Anticonvulsant drugs and mental symptoms: a review. Psychol Med 1976; 169-78.

3 Richens A. Drug treatment of epilepsy. London: Henry Kimpton, 1975.

4 Baloh RW, Konrad HR, Sills AW, Honrubia W. The saccade velocity test. Neurology (Minneapolis) 1975; 25:1071-6.

5 Gresty M, Leech J, Trinder E. Perception of everyday visual environments during saccadic eye movements. A viat Space Environ Med 1976; 47:991-2.

6 Gresty M. The coordination of the head and eyes in pursuit of random and predictable target motion. Aviat Space Environ Med 1977; 48:741-4.

7 Halmagy GM, Gresty MA. Clinical signs of visual-vestibular interaction. $J$ Neurol Neurosurg Psychiatry 1979; 42:934-9.

8 Dichgans J, Von Reutern GM, Rommelt U. Impaired suppression of vestibular nystagmus in cerebellar and non-cerebellar patients. Arch Psychiat NervKrankh 1978; 42:183-99.

9 Benson AJ, Barnes GR. Vision during angular oscillation: the dynamic interaction of visual and vestibular mechanisms. Aviat Space Environ Med 1978; 49:340-5.

10 Baloh RW, Kumley WE, Sills AW, Honrubia V, Konrad HR. Quantitative measurement of smooth-pursuit eye movements. Ann Otol Rhinol Laryngol 1976; 85:111-9.

11 Holzman PS, Proctor LR, Hughes DW. Eyetracking patterns in schizophrenia. Science 1973; 181:179-81.
12 Shagass C, Roemer RA, Amadeo M. Eyetracking performance and engagement of attention. Arch Gen Psychiatry 1976; 33:121-5.

13 Umeda Y, Sakata E. Equilibrium disorders in carbamazepine toxicity. Ann Otol Rhinol Laryngol 1977; 86:318-22.

14 Babin RW. Dilantin suppression. Ann Otol Rhinol Laryngol 1978; 87:284-5.

15 Franck MC, Kuhlo W. Die Wirkung des Alkohols auf die raschen Blickzielbewegungen beim Menschem. Arch Psychiat Nerv Krankh 1970; 213: 238-45.

16 Rashbass C. The relationship between saccadic and smooth-tracking eye movements. J Physiol $1961 ; 159: 326-38$

17 Norris $H$. The action of sedatives on brainstem oculomotor systems in man. Neuropharmacology 1971; 10:181-91.

18 Baloh RW, Honrubia V, Sills A. Eye tracking and optokinetic nystagmus, results of quantitative testing in patients with well-defined nervous system lesions. Ann Otol Rhinol Laryngol 1977, 86:108-14.

19 Hood JD, Korres S. Vestibular suppression in peripheral and central vestibular disorders. Brain 1979; 102:785-804.

20 Norman RN, Sandry S, Corsellis JAN. The nature and origin of patho-anatomical changes in the epileptic brain. In: Vinken PJ, Bruyn GW eds Handbook of Clinical Neurology, Amsterdam: North-Holland Publishing Co 1974; 15:611-20.

21 Salcman M, Defendini R, Correll J, Gilman S. Neuropathological changes in cerebellar biopsies of epileptic patients. Ann Neurol 1978; 3:10-19.

22 Dam M. Dyphenylhydantoin: aspects of toxicity. In: Woodbury DM, Penry JK, Schmidt RP eds. Antiepileptic Drugs. New York: Raven Press 1972; 227-36. 